\title{
Clinical Development and Regulatory Approval of Acute Heart Failure Drugs in Japan
}

\author{
Editorial to: "Rationale and Design of Double-Blind, Randomized, Placebo-Controlled \\ Multicenter Trial on Efficacy of Early Initiation of Eplerenone Treatment in Patients \\ with Acute Heart Failure (EARLIER)" by M. Asakura et al.
}

\author{
Kaori Shinagawa
}

Published online: 14 March 2015

(C) Springer Science+Business Media New York 2015

In this issue of Cardiovascular Drugs and Therapy, Dr. Asakura and co-workers present the design and endpoints of EARLIER, an all-Japanese study with eplerenone in patients with acute, decompensated heart failure (ADHF) [1]. This study is innovative, since it is conducted as an investigatorinitiated clinical trial in Japan to develop a drug that could address an unmet medical need. The study is also interesting in several ways, not the least since the primary objective is to demonstrate benefit in terms of mortality and morbidity of early treatment with an aldosterone antagonist in patients with ADHF; this would represent a new indication for eplerenone, not yet approved in Japan or elsewhere. This strategy is unusual, since clinical studies in Japan more often are conducted to support and confirm results obtained from global clinical trials and to demonstrate that the benefit/risk assessment in the Japanese population supports approval in Japan. It could therefore be of interest to discuss the clinical and regulatory environment for evaluation of drugs for heart failure in Japan.

There are several important aspects that are specific for Japanese heart failure patients, which need to be taken into consideration when developing drugs intended for treatment of this condition in Japan:

- Differences in underlying causes of heart failure:

K. Shinagawa $(\bowtie)$

Office of New Drug II, Pharmaceuticals and Medical Devices

Agency, Shin-kasumigaseki Building, 3-3-2, Kasumigaseki, Chiyoda-ku, Tokyo 100-0013, Japan

e-mail: shinagawa-kaori@pmda.go.jp
Clinical characteristics of Japanese heart failure patients have been assessed trough three epidemiological surveys, the HIJC-HF (Heart Institute of Japan-Department of Cardiology-Heart Failure) [2], the ATTEND (Acute Decompensated Heart Failure Syndromes) [3], and the JCARECARD (Japanese Cardiac Registry of Heart Failure in Cardiology study) [4] registries, evaluating patients hospitalized with exacerbating heart failure. Although comparisons across epidemiological studies in different regions should be done with caution, data obtained in, as an example, the ATTEND Registry can be compared with data from Western countries. It is notable that orthopnea and new onset of ADHF are more common in Japan, even though left ventricular dysfunction is less prevalent and can be estimated to less than half of that of the US population. The incidence of myocardial infarction in Japan is reported to be only one sixth of that in the US, which may at least in part explain this difference. On the other hand, hypertensive heart disease is clearly more prevalent among Japanese heart failure patients and dilated cardiomyopathy is also seen more often. The national surveys have demonstrated that ischemic heart disease accounts for approximately $30 \%$ of heart failure and cardiomyopathy, valvular heart disease, and hypertension account for about $20 \%$ each [2-4].

\section{- Difference in medical practice:}

Guidelines for treatment of heart failure are similar between Japan and Western countries. The Japanese Circulation Society (JCS) developed Guidelines for Treatment of Acute Severe Heart Failure in 2000 [5], and issued Guidelines for Treatment of Acute Heart Failure (JCS2006) in 2006 [6]. 
These guidelines helped to promote standardization of medical care of heart failure patients and in 2011, a partial revision of the guidelines was issued [7]. The guidelines were based on data from Japanese patients, although the AHA (American Heart Association) and ESC (European Society of Cardiology) guidelines were consulted, partially because of insufficient supporting clinical trial data in Japan for some of the covered topics. Japanese medical practice basically follows such guidelines and can therefore be expected to be similar, but not identical to practices in USA and Europe.

In Japan, all patients are covered by a general health insurance, and have free access to health care. This has had a positive influence on the formation of a healthcare environment, which has allowed for standardization of care of heart failure patients and facilitated the implementation of improved therapies, such as early introduction of angiotensin-converting enzyme (ACE) inhibitors and $\beta$-blockers, acute treatment using human atrial natriuretic polypeptide (hANP), common access and use of cardiac rehabilitation, and guidance at discharge based on measured levels of brain natriuretic peptide (BNP). It should be noted that the average duration of the hospital stay for heart failure is 21 days in Japan, which is much longer than in Western countries and the frequency of out-patient visits is also higher in Japan. Mortality is relatively low in Japanese heart failure patients with an in-hospital rate of $7.7 \%$ according to ATTEND registry and all-cause mortality one year after an episode of ADHF of about $10 \%$ according to HIJC-HF and JCARE-CARD, which seems somewhat lower as compared to Europe [8].

Examples of drugs approved in Japan, but not widely used elsewhere for the treatment of heart failure include carperitide (alpha-human atrial natriuretic peptide), tolvaptan (vasopressin V2-receptor antagonist), nicorandil (ATP-sensitive potassium channel opener), and pimobendan ( $\mathrm{Ca} 2+$ sensitizer, $\mathrm{PDE}$ III inhibitor). Carperitide has been marketed for more than a decade in Japan for the treatment of acute heart failure. As intravenous drug therapy during the early phase of hospitalization of decompensated heart failure, diuretics are administered in $80 \%$ of cases, carperitide in $69 \%$, isosorbide dinitrate in $9 \%$, nitroglycerine in $26 \%$ and nicorandil in $11 \%$; inotropic agents are administered in $21 \%$ of cases, which is higher than in the US [3].

\section{- Differences in pharmacodynamic and pharmacokinetics:}

In Japan, the regulatory authority (Pharmaceuticals and Medical Devices Agency, PMDA) usually decides on dose recommendations on the basis of clinical trial data from Japanese subjects. This approach originates at least in part from concerns regarding potential ethnicity-based differences in drug pharmacokinetics (PK) and pharmacodynamics (PD), i.e., which dose is effective in different ethnicities. Indeed, there are many drugs for which the approved doses are different in Caucasian as compared with Japanese patients. For $32 \%(44 / 137)$ of the drugs approved in Japan between 2001 and 2007, the maximum recommended dose was half or less than the corresponding dose in USA [9]. Of all pricelisted cardiovascular drugs currently available in Japan, $65 \%(51 / 79)$ and $57 \%(44 / 77)$ had a maximum recommended dose, which was half or less than the dose in the Netherlands and USA, respectively. These differences in doses across ethnicities are sometimes ascribed to differences in body size and the presence of genetic polymorphisms that affect major clearance pathways, thereby affecting exposure and consequently the drug effect. Examples of this are however relatively few, with rosuvastatin as a notable case in which lower dosage was decided based on the results from a clinical pharmacology study. In many instances, it however seems unlikely that differences in dosing recommendations can be explained on the basis of intrinsic factors alone. Drugs indicated for treatment of heart failure at a lower dose in Japan as compared to Western countries include B-blockers, ACE inhibitors, and angiotensin receptor blockers (ARBs). For example, the dose of carvedilol is recommended to be increased up to $100 \mathrm{mg} /$ day if tolerated in the US and Europe, whereas the maximum approved dose for heart failure is $20 \mathrm{mg}$ /day in Japan. In this context, it should also be emphasized that regional differences exist in regard to how benefit/risk is assessed, which may affect dose selection.

Based on an aging population and improved survival resulting from better treatments, such as ACE inhibitors, the prevalence of heart failure is increasing in Japan. Challenges that meet drug developers for this indication include variability of the underlying heart disease, lack of suitable surrogate endpoints with an acceptable correlation with mortality and difficulties in reaching consensus on the evaluation of subjective endpoint (e.g., dyspnea and quality-of-life, QOL); in fact, few new drugs have been introduced over the last 10 years. To promote research in this therapeutic area of large unmet medical need and to gain further experience based on current medical practice, it is critical to collect data from Japanese patients, either enrolled in global clinical trials or in trials conducted separately in Japan. For this purpose, Ministry of Health, Labour and Welfare (MHLW) issued guidelines in 2011 on the clinical evaluation of drugs for the treatment of heart failure [10]. In these, it is acknowledged that it is difficult to conduct large-scale confirmatory trials with a mortality endpoint in Japan, and other endpoints should therefore be explored in this population. For drugs intended for treatment of acute exacerbations of heart failure, benefits in short-term mortality or morbidity should be demonstrated, without adversely affecting long-term prognosis, i.e., the long-term (more than 6 months) mortality should be at least maintained. Useful endpoints in this setting include subjective and objective symptoms, hemodynamics, and measures of QOL. Only improvement of hemodynamics will not be sufficient; a 
beneficial effect on short-term mortality or improvement of clinical signs and symptoms related to hemodynamics, such as improvement of dyspnea related to pulmonary congestion, must also be demonstrated.

For the treatment of chronic heart failure, an improvement on long-term mortality or improvement in QOL (particularly related to exercise tolerance), again provided long-term mortality is not adversely affected, has to be shown. Because of low mortality rates in Japanese heart failure patients, it is difficult to conduct large-scale confirmatory trial with this endpoint in Japan. In case a mortality benefit is demonstrated in global studies and the results of these studies are applicable to Japan, improvement of morbidity in Japanese patients as evidenced by secondary endpoints may therefore be acceptable for approval. The importance of symptomatic improvement, such as relief of dyspnea or improvement of other QOL measures is well-recognized in Japanese heart failure patients and the identification of appropriate surrogate endpoints, such as biomarkers (BNP), diagnostic imaging, improvement of serious arrhythmias and weight gain/loss as a measure of fluid retention, is encouraged.

Eplerenone is approved in Japan for hypertension but, in contrast to USA and Europe, not yet for treatment of patients with heart failure in general or post-myocardial infarction; the drug is not approved as an acute treatment of ADHF to improve mortality or morbidity in any regulatory jurisdiction. Dr. Asakura and co-workers' study is therefore of large interest. Considering that only a limited number of patients are included in the study, results should be interpreted with appropriate caution. This highlights the importance of meeting the primary endpoint, i.e., demonstrating a beneficial effect on the primary composite endpoint of cardiac death or first rehospitalization due to cardiac disease within 6 months from the enrollment. The results for the secondary endpoints (e.g., incidence of the composite endpoints including cardiac death, first re-hospitalization, and cardiac sudden death, within 1 or 6 months, changes in NYHA cardiac function class from baseline to 1 month or 6 months, heart failure symptom due to KCCQ within 1 month and 6 months, 6 min' walk distance at discharge or in 1 month) should also be evaluated to confirm consistency with the results of primary endpoint. The overall benefit/risk assessment is obviously important in any regulatory discussion, including safety assessment from other studies with eplerenone administered in a similar setting. In addition, the study design including efficacy and safety endpoints need to be evaluated in the context of enabling a proper comparison with the global EPHESUS. Dr. Asakura and coworkers utilized clinical trial consultation meetings with PMDA to discuss the study design including efficacy endpoints prior to initiating the study, and continuous consultation with PMDA from an early stage is beneficial to optimize the development process.

In summary, this study will provide useful information on the effects of eplerenone on acute heart failure in Japanese patients and we all look forward to eventually see the study results presented. It is also rewarding to see how academia, industry and the Japanese government can cooperate to evaluate and hopefully identify better therapies for patients with heart failure.

\section{References}

1. Asakura M, Yamamoto H, Asai K, et al. Rationale and design of double-blind, randomized, placebo-controlled multicenter trial on efficacy of early initiation of eplerenone treatment in patients with acute heart failure (EARLIER). Cardiovasc Drugs Ther 2015;29:this issue.

2. Kawashiro N, Kasanuki H, Ogawa H, Matsuda N, Hagiwara N, Heart Institute of Japan-Department of Cardiology (HIJC) Investigators. Clinical characteristics and outcome of hospitalized patients with congestive heart failure: results of the HIJC-HF registry. Circ J. 2008;72:2015-20.

3. Sato N, Kajimoto K, Asai K, ATTEND Investigators, et al. Acute decompensated heart failure syndromes (ATTEND) registry: A prospective observational multicenter cohort study: Rationale, design, and preliminary data. Am Heart J. 2010;159:949-55.

4. Hamaguchi S, Yokoshiki H, Kinugawa S, Japanese Cardiac Registry of Heart Failure in Cardiology Investigators, et al. Effects of atrial fibrillation on long-term outcomes in patients hospitalized for heart failure in Japan: a report from the Japanese Cardiac Registry of Heart Failure in Cardiology (JCARE-CARD). Circ J. 2009;73:2084-90.

5. Guidelines for the diagnosis and treatment of cardiovascular diseases (1998-1999 Joint Working Groups Report). Guidelines for treatment of severe acute heart failure. Jpn Circ J 2000;64 (Suppl IV):1129-65 (in Japanese).

6. Guidelines for the diagnosis and treatment of cardiovascular diseases (2004-2005 Joint Working Groups Report). Guidelines for treatment of acute heart failure (JCS 2006). http://www.j-circ.or.jp/guideline/ pdf/JCS2006_maruyama_h.pdf (in Japanese).

7. Guidelines for the diagnosis and treatment of cardiovascular diseases (2010 Joint Working Groups Report). Guidelines for Treatment of Acute Heart Failure (JCS 2011). http://www.j-circ.or.jp/guideline/ pdf/JCS2011_izumi_h.pdf (in Japanese).

8. Harjola V-P, Follath F, Nieminen MS, et al. Characteristics, outcomes, and predictors of mortality at 3 months and 1 year in patients hospitalized for acute heart failure. Eur J Heart Fail. 2010;12: $1239-48$.

9. Arnold FL, Kusama M, Ono S. Exploring differences in drug doses between Japan and Western countries. Clin Pharmacol Ther. 2010;87: 714-20.

10. Guidelines for clinical evaluation of heart failure drugs. http://www. pmda.go.jp/kijunsakusei/file/guideline/new drug/koushinfuzenyaku-rinjyu-hyouka-guideline.pdf (in Japanese). 